Z. klin. Chem. u. klin. Biochem.

7. Jg., S. 636-639, November 1969

\title{
Eine schnelle Methode zur Bestimmung des gesamten und proteingebundenen Jods (PBI)
}

\author{
Von D. JÜNGST und L. STRAUCH \\ Max-Planck-Institut fïr Eiveiß- und Lederforschung, Mïnchen
}

(Eingegangen am 31. Juli 1969)

\begin{abstract}
Bei der beschriebenen Methode werden $0,1 \mathrm{~m} /$ einer Serumprobe, die zur Bestimmung von PBI mit Dowex 1 X 8 vorbehandelt wurde, in Reagenzgläsern mit einem $\mathrm{HClO}_{3}$-Reagenz bei $120^{\circ}-160^{\circ}$ aufgeschlossen. Der Aufschluß dauert 30 Min. und wird mit einem temperaturregulierten Thermoblock vorgenommen. Die Jodbestimmung erfolgt durch katalytische Reduktion von Cer(IV)-ionen. Die Reaktion wird durch Zugabe von Brucinsulfat abgestoppt und die Farbintensität des dabei gebildeten Brucin-Cer-Komplexes gemessen. Die Fehlergrenze beträgt unter 3\%. Eine geübte Arbeitskraft kann mit einem Thermoblock täglich etwa 80-100 Serumproben analysieren.
\end{abstract}

\section{$A$ rapid method for the determination of total and protein-bound iodine (PBI)}

In the present method, a $0.1 \mathrm{~m} l$ serum sample, pretreated with Dowex 1 X 8 for the determination of PBI, is digested in a test tube with a $\mathrm{HClO}_{3}$ reagent at $120^{\circ}-160^{\circ}$. The digestion lasts $30 \mathrm{~min}$. and is performed in a temperature-regulated thermoblock. The iodine is determined by the catalytic reduction of $\operatorname{Cer}(\mathrm{IV})$-ions. The reaction is stopped by the addition of brucine sulphate and the colour intensity of the resulting brucine-Cer complex is measured. The error is less than $3 \%$. With the aid of a thermoblock and with previous experience, $80-100$ samples per day may be analysed.

Die Grundlagen der Schilddrüsendiagnostik beruhen auf den engen Beziehungen, die zwischen Jodstoffwechsel und Schilddrüsenaktivität bestehen. Das proteingebundene Jod (PBI) im Serum besteht zum größten Teil aus dem in L-Thyroxin enthaltenen Jod. Die Menge des PBI gibt uns daher einen direkten Einblick in die Funktion der Schilddrüse.

Die Jodkonzentration eines normalen Humanserums liegt im Bereich von $3,2-7,2 \mu \mathrm{g} / 100 \mathrm{~m} l$. Sie entzieht sich aus diesem Grund einem direkten chemischen Nachweis und ist nur mit Hilfe einer katalytischen Reaktion zu erfassen.

Die Bestimmung von PBI umfaßt im wesentlichen drei Schritte :

Trennung des PBI von anorganischem Jod, die durch Ausfällung von Serumproteinen $(1,2)$ oder durch Ausschütteln des Serums mit Ionenaustauschern erreicht werden kann $(3,4,5)$.

Einveißaufscbluß und Isolierung des gebundenen Jods in der für die Bestimmung notwendigen Form. Von den trockenen Veraschungsmethoden (6) ist man in der letzten Zeit auf nasse Veraschungen übergegangen, von denen alkalische Aufschlüsse (7), saure Verfahren mit $\mathrm{HClO}_{3}(2,4,8)$ oder mit $\mathrm{HClO}_{4}$ und $\mathrm{HNO}_{3}(3,5,9)$ mit gutem Erfolg angewendet werden.

Kolorimetrische Bestimmung mit Hilfe einer katalytischen Reaktion. In neuerer Zeit hat sich zur Bestimmung kleinerer Jodmengen ausschließlich das erstmalig von Sandell und Kolthoff (10) verwendete Prinzip der katalytischen Beschleunigung der Reaktion

$$
2 \mathrm{Ce}^{4+}+\mathrm{As}^{3+} \longleftrightarrow 2 \mathrm{Ce}^{3+}+\mathrm{As}^{5+}
$$

durch Jod durchgesetzt.
Die hier beschriebene Methode ist durch ihre Einfachheit und den geringen apparativen Aufwand jedem Krankenhauslaboratorium zugänglich und kann mit Erfolg für Routineuntersuchungen eingesetzt werden. Das anorganische Jod wird an Ionenaustauscher gebunden und so von PBI abgetrennt. Der Aufschluß der organischen Substanz und die Oxydation des Jods zum Jodat wird mit $\mathrm{HClO}_{3}$ und Natriumchromat durchgeführt, da mit diesem Verfahren auch kleinste Jodmengen im Bereich von $1 \mathrm{ng}$ mit Sicherheit erfaßt werden können. Im Gegensatz zu anderen Methoden, die zur Erhitzung des Aufschlußgemisches Kochplatten (2), Ölbäder (11) oder Sandbäder (12) verwenden, wird der Aufschluß hier in einem temperaturregulierbaren Thermoblock vorgenommen. Die kolorimetrische Jodbestimmung erfolgt nach der oben angegebenen Reaktion, wobei die durch Jod katalysierte Reduktion des gelben $\mathrm{Ce}^{4+} \mathrm{zu}$ farblosem $\mathrm{Ce}^{3+}$ durch Zugabe von Brucinsulfat unterbrochen wird $(4,13-16)$.

\section{Methodik}

\section{Reagenzien}

Es werden Chemikalien des Reinheitsgrades pro analysi sowie deionisiertes Wasser mit einer Leitfähigkeit unter $0,1 \mu \mathrm{S}$ verwendet.

Ionenaustauscher: Dowex 1 X 8, 20-50 mesh, Cl-Form. $\mathrm{HClO}_{3}$-Reagenz nach $\mathrm{ZAK}(2)$ :

$50 \mathrm{~g} \mathrm{KClO}_{3}$ werden in $100 \mathrm{~m} /$ Wasser aufgelöst und erhitzt. Zur siedenden klaren Lösung werden langsam $37 \mathrm{~m} / \mathrm{HClO}_{4}$ (70 proz. Dichte 1,67) gegeben. Anschließend wird die Lösung etwa 8 Stdn. bei $+4^{\circ}$ aufbewahrt, wodurch der gẹbildete $\mathrm{KClO}_{4}$-Niederschlag vollständig auskristallisiert. Nach Abfiltrieren wird das Aufschlußreagenz, etwa 30 proz. $\mathrm{HClO}_{3}$, bei $+4^{\circ}$ in einer dunklen Flasche aufbewahrt. 
Natriumchromatlösung 0,6proz. (g/v) in Wasser: $0,6 \mathrm{~g} \mathrm{Na}_{2} \mathrm{CrO}_{4}$ werden in $100 \mathrm{~m} /$ Wasser aufgelöst.

Konz. $\mathrm{H}_{2} \mathrm{SO}_{4}$ (Dichte 1,84).

Arsenige Säure $\left(0,2 \mathrm{~N}\right.$ in $\left.1 \mathrm{~N}_{2} \mathrm{SO}_{4}\right)$ :

$1,96 \mathrm{~g} \mathrm{As}_{2} \mathrm{O}_{3}$ und $1,4 \mathrm{~g} \mathrm{NaOH}$-Plätzchen werden in $80 \mathrm{~m} /$ Wasser aufgelöst. Mit Phenolphthalein wird mit konz. Schwefelsäure bis zum Umschlagspunkt titriert. Nach Zugabe von $5,6 \mathrm{ml}$ konz. $\mathrm{H}_{2} \mathrm{SO}_{4}$ wird auf $200 \mathrm{~m} /$ aufgefüllt. Anschließend werden $5,0 \mathrm{~g}$ $\mathrm{NaCl}$ dazugegeben und aufgelöst.

Cerammoniumsulfat $\left(0,025 \mathrm{~N}\right.$ in $\left.2 \mathrm{~N} \mathrm{H}_{2} \mathrm{SO}_{4}\right)$ :

$1,57 \mathrm{~g}$ Cerammoniumsulfat und $5,2 \mathrm{~m} / \mathrm{konz} . \mathrm{H}_{2} \mathrm{SO}_{4}$ werden in $100 \mathrm{~m} /$ Wasser aufgelöst.

Brucinsulfat 1,0 proz. (g/v):

$1,0 \mathrm{~g}$ Brucinsulfat werden in $100 \mathrm{~m} /$ Wasser aufgelöst.

Jodstandardlösungen:

$168,5 \mathrm{mg} \mathrm{KJO} \mathrm{KJ}_{3}$ werden in $1 \mathrm{~m} /$ Wasser aufgelöst $(100 \mu \mathrm{g} \mathrm{Jod} / \mathrm{m} /)$. Von dieser Stammlösung wird durch mehrmaliges Verdünnen eine $16 \mu \mathrm{g} \mathrm{Jod} / 100 \mathrm{ml}$-Lösung hergestcllt.

\section{Apparaturen}

\section{Glasgeräte}

Für die Bestimmung werden Reagenzgläser $16 \times 160 \mathrm{~mm}$ Duran 50, Schott u. Gen., Mainz, verwendet. Weiterhin werden Meßpipetten von $0,1-1,0 \mathrm{~m} l$ sowie Vollpipetten von 1,0 und $2,0 \mathrm{~m} /$ benötigt. Auf eine gründliche Reinigung aller bei der PBI-Bestimmung benutzten Glasgeräte muß besonderer Wert gelegt werden.

\section{Aufscblußgerät}

Der Aufschluß erfolgt im WTW-Thermoblock (WissenschaftlichTechnische Werkstätten GmbH, 812 Weilheim/Obb., Trifthofstr., zu beziehen unter der Bezeichnung Thermoblock TB 4), einem elektrisch beheizten Aluminiumblock, der mit einem stufenlos einstellbaren Temperaturregler ausgestattet ist. Zur besseren Erwärmung der aus dem Gerät ragenden Reagenzgläser wird auf den Block ein außen isolierter Blechring aufgesetzt.

\section{Photometer}

Die optischen Messungen wurden in der vorliegenden Arbeit mit einem Beckman DU Spektrophotometer ausgeführt.

\section{Ausführung der Bestimmung}

\section{Abtrennung des anorganiscben Jods}

$1,5-2,0 \mathrm{~m} /$ Serum werden in einem trockenen Reagenzglas mit $300 \mathrm{mg}$ Ionenaustauscher versetzt und etwa $5 \mathrm{Min}$. ausgeschüttelt.

\section{Aufscblup der organiscben Substanz}

Nach Absetzen des Ionenaustauschers werden vom Überstand $0,1 \mathrm{~m} /$ Serum entnommen und in ein Reagenzglas gegeben. Für diesen Arbeitsgang empfiehlt sich die Anwendung einer automatischen Eppendorfpipette mit auswechselbarer Kunststoffspitze. Nach Zugabe von $1,0 \mathrm{~m} / \mathrm{HClO}_{3}$-Reagenz und $0,05 \mathrm{~m} / 0,6 \mathrm{proz}$. $\mathrm{Na}_{2} \mathrm{CrO}_{4}$-Lösung werden die Reagenzgläser in die Bohrungen des auf $120^{\circ}$ vorgewärmten Thermoblocks mit dem aufgesetzten Wärmedämmring eingesetzt. Ein Schütteln der Reagenzgläser ist unbedingt $\mathrm{zu}$ vermeiden, damit der Proteinniederschlag nicht $\mathrm{zu}$ hoch an der Glaswand haften bleibt und so der Einwirkung des beißen Aufschlußgemisches entzogen wird. Analog zur unbekannten Serumprobe wird von der $16 \mu \mathrm{g} / 100 \mathrm{ml}$ Jodstandardlösung eine Verdünnungsreihe mit $0 \mathrm{~m} l ; 0,025 \mathrm{~m} / ; 0,05 \mathrm{~m} / ; 0,075 \mathrm{~m} l$; $0,1 \mathrm{ml}$ cntsprechend $0 ; 4,0 ; 8,0 ; 12,0 ; 16,0 \mu \mathrm{g} / \mathrm{Jod} / 100 \mathrm{ml}$ angesetzt. Nach Ablauf der ersten $10 \mathrm{Min}$. wird die Aufschlußtemperatur von $120^{\circ}$ auf $160^{\circ}$ erhöht. Die gebildete $\mathrm{HClO}_{4}$ steigt in Form eincs Siederinges, der aus kleinen, das Glas nicht benetzenden Tröpfehen besteht, die Glaswand hoch. Innerhalb von etwa 5 Min. ist die Endtemperatur von $160^{\circ}$ erreicht, bei der der Aufschluß noch weitere 15 Min. geführt wird. Die gesamte Aufschlußdauer beträgt also etwa $30 \mathrm{Min}$. Am Ende des Aufschlusses verbleibt cin bernsteinfarbener Rückstand von etwa $0,1 \mathrm{~m} /$ Volumen. Die Reagenzgläser werden aus dem Thermoblock entfernt und auf Zimmertemperatur abgckühlt, wobei rote $\mathrm{CrO}_{3}$ - $\mathrm{Kristallc}$ ausfallen.

\section{Die kolorimetrische Bestimmung}

$\mathrm{Zu}$ dem in den Reagenzgläsern verbliebenen Rückstand werden $0,2 \mathrm{~m} / \mathrm{konz} . \mathrm{H}_{2} \mathrm{SO}_{4}$ sowie $2,0 \mathrm{~m} /$ arsenige Säure gegeben. Um eine vollständige Reduktion des Jodats in Jodid zu erreichen, werden die Proben 20 Min. im Wasserbad bei $37^{\circ}$ inkubiert (15). In genauen Zeitabständen (20-30 Sek.) wird $1,0 \mathrm{~m} /$ Cerammoniumsulfatlösung hinzugefügt und sofort gut umgeschüttelt. Nach einer bestimmten Zeit, die für alle Proben genau gleich sein muß (in der Praxis zwischen 20 bis 30 Min.), wird die Farbreaktion durch Zugabe von $0,2 \mathrm{~m} /$ Brucinsulfatlösung unterbrochen. Um eine Stabilisierung des Brucin-Cer-Komplexes zu erreichen, werden die Proben etwa $30 \mathrm{Min}$. bei $37^{\circ}$ inkubiert $(15,16)$. Die Messung der Farbintensität erfolgt bei $428 \mathrm{~nm}$.

Aus den Meßergebnissen der Jodstandardreihe wird auf semilogarithmischem Millimeterpapier ein Meßdiagramm konstruiert und aus diesem der Gehalt der unbekannten PBI-Mengen ermittelt.

\section{Ergebnisse}

Um die Vollständigkeit des Aufschlusses zu überprüfen, wurden exakt eingewogene organische jodhaltige Testsubstanzen untersucht.

Tab. 1

Bestimmung des Jodgehaltes in Testsubstanzen

\begin{tabular}{lccr}
\hline \multicolumn{1}{c}{ Probe } & Jod vorgegeben & \multicolumn{2}{c}{ Jod gefunden } \\
& $(\mu \mathrm{g})$ & $(\mu \mathrm{g})$ & $(\%)$ \\
\hline $\begin{array}{l}\text { Jodacetamid gelöst } \\
\text { in } \mathrm{H}_{2} \mathrm{O}\end{array}$ & 0,108 & 0,111 & 102,8 \\
& & 0,120 & 111,1 \\
& & 0,116 & 107,4 \\
Jodessigsäure & 0,147 & 0,119 & 110,4 \\
& & 0,132 & 89,8 \\
& & 0,143 & 97,3 \\
& & 0,150 & 102,0 \\
Dijodtyrosin gelöst in & 0,054 & 0,153 & 104,1 \\
verd. $\mathrm{H}_{2} \mathrm{SO}_{4}$ & & 0,060 & 111,1 \\
L-Thyroxin gelöst in & 0,034 & 0,056 & 103,7 \\
verd. $\mathrm{NaOH}$ & & 0,033 & 97,1 \\
\hline
\end{tabular}

Um Jodverluste während des Aufschlusses auszuschlieBen wurden Seren mit bekanntem Jodgehalt mit zugefügten $\mathrm{KJO}_{3}$-Standatds und mit Testsubstanzen untersucht.

Tab. 2

Bestimmung des Jodgehaltes im Serum unter Zugabe von Testsubstanzen

\begin{tabular}{|c|c|c|c|c|}
\hline \multirow{2}{*}{$\begin{array}{l}\text { Serum-Jod } \\
\frac{(\mu \mathrm{g} / 100 \mathrm{ml})}{3,0}\end{array}$} & \multirow{2}{*}{$\frac{\begin{array}{c}\text { Jod } \\
\text { hinzugefügt } \\
(\mu \mathrm{g} / 100 \mathrm{ml})\end{array}}{4,0}$} & \multirow{2}{*}{$\frac{\text { als }}{\mathrm{KJO}_{3}}$} & \multicolumn{2}{|c|}{$\underset{(\mu \mathrm{g} / 100 \mathrm{~m} l)}{\text { berechnet }} \stackrel{\text { gefunden }}{ }$} \\
\hline & & & 7,0 & $\begin{array}{l}6,8 \\
6,6 \\
7,1 \\
7,3\end{array}$ \\
\hline 5,0 & 4,0 & $\mathrm{KJO}_{3}$ & 9,0 & $\begin{array}{l}8,2 \\
8,5 \\
8,8 \\
9,3\end{array}$ \\
\hline 5,0 & 8,0 & $\mathrm{KJO}_{3}$ & 13,0 & $\begin{array}{l}12,3 \\
12,8 \\
13,2 \\
13,4\end{array}$ \\
\hline 3,0 & 3,3 & Thyroxin & 6,3 & $\begin{array}{l}6,0 \\
6,4\end{array}$ \\
\hline 3,0 & 5,4 & Dijodtyrosin & 8,4 & $\begin{array}{l}8,5 \\
9,0\end{array}$ \\
\hline 3,0 & 10,8 & Jodacetamid & 13,8 & $\begin{array}{l}13,5 \\
13,5\end{array}$ \\
\hline 3,0 & 14,7 & Jodessigsäure & 17,7 & $\begin{array}{l}17,0 \\
17,7\end{array}$ \\
\hline
\end{tabular}


Zur Festlegung der Fehlergrenze wurde ein Humanserum in mehreren Analysen untersucht.

Tab. 3

Bestimmung des Jodgehaltes in einem humanen Standardserum

\begin{tabular}{ccc}
\hline $\begin{array}{c}\text { Probe } \\
\text { lfd. Nr. }\end{array}$ & $\begin{array}{c}\text { Jod gefunden } \\
(\mu \mathrm{g} / 100 \mathrm{ml})\end{array}$ & $\begin{array}{c}\text { \% des arith- } \\
\text { metrischen Mittels }\end{array}$ \\
\hline 1. & 5,5 & 94,4 \\
2. & 5,7 & 97,9 \\
3. & 6,0 & 103,0 \\
4. & 5,7 & 97,9 \\
5. & 5,9 & 101,3 \\
6. & 5,9 & $-101,3$ \\
7. & 5,8 & 99,5 \\
8. & 5,8 & 99,5 \\
9. & 5,6 & 96,1 \\
10. & 6,1 & 104,7 \\
11. & 5,6 & 103,0 \\
12. & 5,9 & 96,1 \\
13. & 5,8 & 101,3 \\
14. & 6,0 & 99,5 \\
15. & 6,1 & 103,0 \\
16. & 5,8 & 104,7 \\
17. & 5,9 & 99,5 \\
18. & 5,6 & 101,3 \\
19. & 5,8 & 96,1 \\
20. & $-325 \pm 0,17$ & $100,0 \pm 3,0$ \\
\hline $\bar{x}$ & 5
\end{tabular}

Die an Hand der Tabelle 3 ausgerechnete Standardabweichung beträgt $\pm 0,17$ oder weniger als $3 \%$ des Mittelwertes.

Eine typische Eichgerade zur Bestimmung der unbekannten Jodkonzentration ist in Abbildung 1 angegeben.

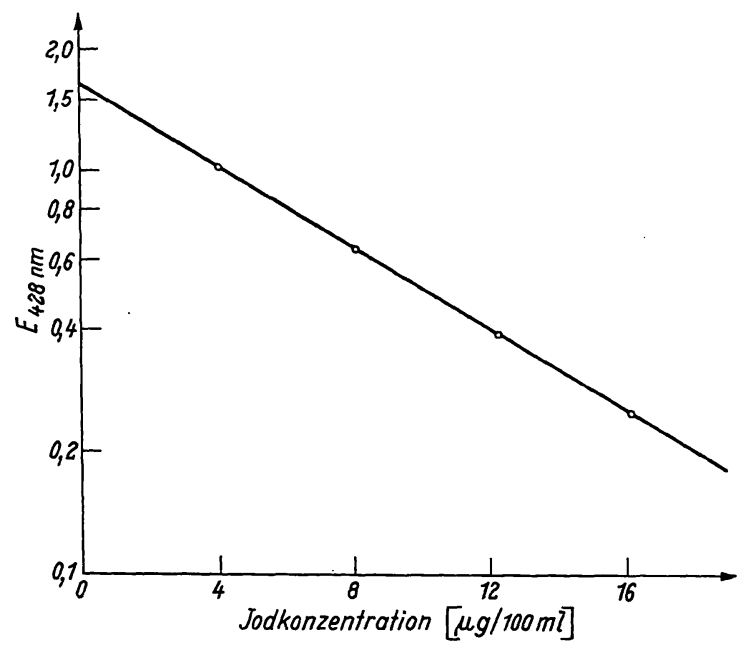

Abb. 1

Semilogarithmische Auftragung der Jodkonzentration in Abhängigkeit von der Farbintensität des Brucin-Cer-Komplexes

\section{Diskussion}

Die beschriebene Methode stellt eine Verbesserung der von ZAK (2) entwickelten Jodbestimmung dar. Das anorganische Jod im Serum wird mit Ionenaustauschern abgetrennt, wodurch die Bestimmung erheblich verkürzt und vereinfacht wird. Mit radioaktiven Jodisotopen konnte nachgewiesen werden, daß über $90 \%$ des anorganischen Jods an den Ionenaustauscher adsorbiert werden (3). Der anorganische Anteil am Gesamtjod im Serum beträgt etwa $10 \%$, so daß er nach der Behandlung mit dem Ionenaustauscher auf etwa 1\% zurückgeht. Der Aufschluß der organischen Substanz wird bei Temperaturen von $120-160^{\circ}$ durchgeführt. Die während des Aufschlusses notwendige Temperaturregelung ist bei Verwendung des Thermoblocks einfach zu verwirklichen. Die Veraschungsdauer hängt im wesentlichen von der Menge des verwendeten $1 \mathrm{HClO}_{3}$-Reagenzes ab. Um ein ausreichendes Sauerstoffangebot zu gewährleisten, sollte das Verhältnis $\mathrm{HClO}_{3}$ : Serum etwa 10:1 betragen. In der angegebenen Methode werden $0,1 \mathrm{ml}$ Serum mit $1,0 \mathrm{ml} \mathrm{HClO}_{3}$-Reagenz aufgeschlossen, wodurch die Serumproben schon nach $30 \mathrm{Min}$. ausgewertet werden können. Die Anwesenheit von $\mathrm{Na}_{2} \mathrm{CrO}_{4}$ hat auf das Oxydationspotential keinen Einfluß. Die Substanz dient als Redox-Indikator, da die Chromionen bei verschiedenen Oxydationsstufen verschiedene Farben annehmen (17). Gelb gefärbte $\mathrm{CrO}_{3}$-Ionen zeigen an, $\mathrm{da} B$ das vorhandene Jod mit Sicherheit in der nichtflüchtigen $\mathrm{HJO}_{3}$-Form vorliegt. Schlägt die Farbe in irgendeiner Phase des Aufschlusses in grün, als Anzeichen von $\mathrm{Cr}_{2} \mathrm{O}_{3}$-Ionen um, sind Jodverluste durch Bildung von gasförmigen $\mathrm{HJ}$ möglich.

Das Auftreten von grünen Chromionen beobachtet man am häufigsten in der zweiten Hälfte des Aufschlusses, wenn nur noch geringe Mengen $\mathrm{HClO}_{3}$ vorhanden sind. Durch Zugabe von einem Tropfen Aufschlußreagenz $10 \mathrm{Min}$. vor Ende des Aufschlusses kann diese Erscheinung verhindert werden. Die gleichmäßige Erwärmung der Reagenzgläser durch den Wärmedämmring ermöglicht eine schnelle Aufschlußführung, da die Bildung von Kondenswasser an der Glaswand verhindert wird. Außerdem wird dadurch die Kondensation von niederen Fettsäuren oder anderer unvollständiger Oxydationsprodukte des Serums an der Glaswand verhindert (8). Schon Spuren dieser Substanzen interferieren mit der anschließenden kolorimetrischen Bestimmung, da sie die Entfärbung des Cerions durch direkte Reduktion beschleunigen.

Am Ende des Aufschlusses muß auf annähernde Volumengleichheit der verbliebenen $\mathrm{HClO}_{4}$-Chromat-Rückstände geachtet werden, damit bei der kolorimetrischen Bestimmung gleiche $\mathrm{HClO}_{4}$-Konzentrationen vorliegen.

Die Vollständigkeit des Aufschlusses muß nach drei Gesichtspunkten beurteilt werden:

a) Als Zeichen für die vollständige Umwandlung der $\mathrm{HClO}_{3}$ in $\mathrm{HClO}_{4}$ gilt das Auftreten von $\mathrm{HClO}_{4}^{-}$ Tröpfchen, die die Glaswand nicht benetzen. Dieser Punkt muß unbedingt erfüllt sein, da zurückgebliebene $\mathrm{HClO}_{3}$ durch Reoxydierung des gebildeten $\mathrm{Cer}^{3+}$-Ions mit der kolorimetrischen Bestimmung interferiert. Außerdem bildet sie mit Brucin einen gelben Farbstoff, der ein Abstoppen der Reaktion unmöglich machen würde (16).

b) Nach Abkühlung des Aufschlußgemisches auf Raumtemperatur müssen rote $\mathrm{CrO}_{3}$-Kristalle ausfallen.

c) Nach Absetzen der Kristalle muß ein klarer und farbloser Überstand entstehen. :

Diese Bedingungen sind bei Einhaltung der vorgeschriebenen Arbeitsweise in den meisten Fällen gegeben. 
Die kolorimetrische Bestimmung wird im gleichen Reagenzglas durchgeführt, so daß Verunreinigungen und Substanzverluste vermieden werden. Wegen der geringen aufgeschlossenen Serummengen liegen die zu bestimmenden Jodmengen im Bereich von 0-16 ng. Es ist bezeichnend für die Empfindlichkeit der Farbreaktion, daß sich solche Mengen noch exakt bestimmen lassen. Der katalytische Effekt des Jodidions kommt besonders gut zur Geltung, wenn die im Reaktionsgemisch vorliegenden $\mathrm{H}_{2} \mathrm{SO}_{4}^{-}$und $\mathrm{HClO}_{4}$-Konzentrationen in einem günstigen Verhältnis stehen. Während eine Erhöhung der $\mathrm{H}_{2} \mathrm{SO}_{4}$-Konzentration die Entfärbung des $\mathrm{Cer}^{4+}$-Ions verlangsamt und damit die $\mathrm{Em}$ pfindlichkeit der kolorimetrischen Bestimmung erhöht, macht sich bei einer Erhöhung der $\mathrm{HClO}_{4}^{-}$ Konzentration der umgekehrte Effekt bemerkbar. Die exaktesten und steilsten Standardgeraden erzielt man bei einer 4-8 $\mathrm{N} \mathrm{H}_{2} \mathrm{SO}_{4}$-Konzentration und möglichst geringem $\mathrm{HClO}_{4}-$ Gehalt. Diese Bedingungen sind in der beschriebenen Methode erfüllt (18).
Die Unterbrechung der Farbreaktion ermöglicht eine exakte Auswertung, da sich die Intensität des stabilisierten Brucin-Cer-Komplexes innerhalb von 24 Stdn. nicht ändert (16).

Die mit dieser Methode erzielten Ergebnisse zeigen eine gute Reproduzierbarkeit der Standardgeraden. Die gegebenen jodhaltigen Testsubstanzen wurden vollständig wiedergefunden. Bei der Bestimmung von Jodstandards und von Testsubstanzen im Serum konnten keine Jodverluste beobachtet werden. Die aus einer Mehrfachbestimmung des Humanserumpools ermittelte Standardabweichung liegt noch unter $\pm 3 \%$ des Mittelwertes.

Eine eingeübte Arbeitskraft kann in einem Arbeitsgang, der etwa 2 Stdn. dauert, mit einem Aufschlußgerät 10 Serumproben in Parallelbestimmung analysieren. Die beschriebene Methode eignet sich sehr gut zur Routinebestimmung von PBI sowie von Gesamtjod und kann, wegen des geringen apparativen Aufwandes, auch in kleineren Laboratorien ausgeführt werden.

\section{Literatur}

1. Somogyi, M., J. biol. Chemistry 86, 655 (1930). - 2. ZAK, B., H. H. Willard, G. B. Myers und A. J. Boyle, Analytic. Chem. 24, 1345 (1952). - 3. Kutzim, H., Technicon Symposion 1965, Frankfurt, S. 855. - 4. FARrell, L. P. und M. H. Ricimond, Clin. chimica Acta Amsterdam 6, 620 (1961). - 5. Technicon Instruction Manual, NO. PB/D - 1. - 6. MrcheL, O. und G. Deltour, Buli. Soc. Chim. biol. 31, 1125 (1949). - 7. BARKER, S. B., J. biol. Chemistry 173, 715 (1948). - 8. Hoch, H., S. L. Sinnett und T. H. McGavaCk, Clin. Chem. New York 10, No. 9 (1964). - 9. Gochman, N., Technicon Symposion 1964, Frankfurt, S. 429. - 10. SANDELL, E. B. und J. M. Kolthoff,
Mikrochim. Acta 1, 9 (1937). - 11. Mantzos, J. D. und B. Malamos, Clin. chimica Acta Amsterdam 21, 501 (1968). - 12. Bodansky, O., R. S. Benua und G. Pennacchia, Amer. J. Clin. Path. 30, 375 (1958). - 13. FischL, J., Clin. chimica Acta Amsterdam 1, 462 (1956). - 14. Grossmann, A. und G. F. Grossmann, J. Clin. Endocr. Springfield 15, 354 (1955). - 15. Lepp, A., H. Pena, V. Hoxie und L. Oliner, Amer. J. Clin. Path. 44, 331 (1965). 16. Haschen, R. J. und N. Rehfeld, Dtsch. Gesd.wes. 37, 1590 (1963). - 17. StrickLand, R. D., und C. M. Maloney, Analytic Chem. 29, 1870 (1957). - 18. JüNGST, D., Dissertation, Universität München, 1969.
Dr. L. Strauch 8000 München 15 Schillerstr. 46 